\title{
Long-term outcomes after aortic root replacement for patients with Marfan syndrome
}

\author{
Yu Zhu^, Zhao Jian, Ruiyan Ma, Yong Wang, Yingbin Xiao^ \\ Department of Cardiovascular Surgery, the Second Affiliated Hospital, Army Medical University, Chongqing, China \\ Contributions: (I) Conception and design: Y Zhu, Y Xiao; (II) Administrative support: Y Xiao; (III) Provision of study materials or patients: \\ All authors; (IV) Collection and assembly of data: All authors; (V) Data analysis and interpretation: Y Zhu, Z Jian; (VI) Manuscript writing: \\ All authors; (VII) Final approval of manuscript: All authors. \\ Correspondence to: Yingbin Xiao; Zhao Jian. Department of Cardiovascular Surgery, the Second Affiliated Hospital, Army Medical University, \\ Chongqing 400037, China. Email: xiaoyb@tmmu.edu.cn; zhao.j@tmmu.edu.cn.
}

Background: A diversity of surgical strategies are used to treat Marfan syndrome patients with aortic disease. We sought to evaluate the long-term efficiency of aortic root replacement (ARR) for patients with Marfan syndrome.

Methods: Data were collected from 131 patients with Marfan syndrome and aortic disease who underwent ARR in our center. We retrospectively analyzed the long-term outcomes of these patients, among whom 68 had been diagnosed with aortic aneurysm (AA) and had undergone ARR. The remaining 63 patients had aortic dissection (AD); of these, 35 underwent ARR for limited ascending AD, while the others underwent ARR and total arch replacement combined with frozen elephant trunk (FET). Risk factors for survival and reoperation were identified.

Results: The operative mortality rate was $4.58 \%$. Age $>40$ years was the sole risk factor for operative mortality. During follow-up, 12 deaths occurred. Patients aged $<25$ years and female patients were more prone to late death than were other patients. Indications for reoperation were noted in 22 patients, and the risk factors were age $<30$ years and female sex. At 5 years, the survival rate was $92.96 \%$, and the freedom from reoperation rate was $88.36 \%$. At 10 years, the survival rate was $85.25 \%$, and the freedom from reoperation rate was $71.75 \%$. The survival and freedom from reoperation rates were significantly higher in patients with $\mathrm{AD}$ than in those with $\mathrm{AA}$. Specifically, chronic $\mathrm{AD}$ was a greater risk factor for late survival than was acute $\mathrm{AD}$ in patients with Marfan syndrome.

Conclusions: For patients with Marfan syndrome and aortic disease, ARR can be safely performed and results in low operative mortality and favorable long-term survival. Young and female patients have a higher risk for late death and reoperation. To prevent $\mathrm{AD}$, surgical intervention should be promptly implemented following the diagnosis of aortic sinus dilation.

Keywords: Aortic root replacement (ARR); Marfan syndrome; long-term outcomes

Submitted Apr 02, 2021. Accepted for publication Oct 22, 2021.

doi: $10.21037 /$ jtd-21-577

View this article at: https://dx.doi.org/10.21037/jtd-21-577

^ ORCID: Yu Zhu, 0000-0003-1133-8376; Yingbin Xiao, 0000-0003-1854-8982. 


\section{Introduction}

Marfan syndrome is the most common cause of syndromic ascending aortic aneurysm (AA), with an average expansion rate of approximately $0.5-1 \mathrm{~mm}$ per year (1). Clinically, replacement of the dilated aortic root, including aortic valve cusps (Bentall procedure) or valve sparing root surgery (David procedure), has been the standard treatment for aortic root aneurysms (2-5) and can effectively improve the long-term prognosis. Thoracic and abdominal aorta replacement and frozen elephant trunk (FET) are also efficacious strategies for patients with distal dissection or aneurysms. However, surgical intervention does not always completely resolve the issue due to the physiopathology of Marfan syndrome and guidelines do not specify how to select a surgical procedure in patients with different types of Marfan syndrome. Hence, we reviewed the experiences of our team in the treatment of patients with Marfan syndrome, aiming to evaluate the long-term efficacy of aortic root replacement (ARR) in our department and to provide evidence on treatment strategies for patients with Marfan syndrome.

We present the following article in accordance with the STROBE reporting checklist (available at https://dx.doi. org/10.21037/jtd-21-577).

\section{Methods}

\section{Patients}

The study was conducted in accordance with the Declaration of Helsinki (as revised in 2013). The retrospective study was approved by the Medical Ethics Committee of the Second Affiliated Hospital, Army Medical University, Chongqing, China (No. 2020-Yan-122-01) and informed consent was taken from all the patients. Between January 2002 and December 2017, our team operated on 131 patients with Marfan syndrome and aortic disease, among whom 68 patients were diagnosed with AA and underwent ARR. The remaining 63 patients had aortic dissection (AD); 35 underwent ARR because the dissection originated in and was confined to the ascending aorta, and the others underwent ARR and total arch replacement combined with FET. The diagnosis of Marfan syndrome was confirmed preoperatively according to the Ghent criteria and/or revised Ghent criteria based on physical examination findings (6). Patients were excluded from the study if rupture of the dissection occurred before surgery. All cases were retrospectively reviewed using a common research protocol. The following elements were included: (I) baseline patient characteristics and preoperative clinical profiles; (II) intraoperative data; (III) postoperative data and operative mortality; (IV) long-term follow-up data; and (V) late death and reoperations.

\section{Indication for surgery and surgical techniques}

In Marfan syndrome patients presenting with aortic valve regurgitation (AR), ARR (Bentall procedure) was performed with mechanical prosthesis. In those presenting with Stanford type A acute AD, the procedures of ARR and total arch replacement with FET were performed.

The surgical technique has been previously described in detail (7-9). Briefly, general anesthesia was induced in the supine position. A standard median sternotomy was performed in all patients. The right axillary artery, right atrial, and/or femoral artery were cannulated for the establishment of a cardiopulmonary bypass (CPB). For patients complicated with mitral or tricuspid valve surgery, inferior and superior bicaval cannulae were used. The ascending aorta was cross-clamped, and cold blood cardioplegia was infused via the coronary ostia for myocardial protection. Bentall procedure was performed for participants with aortic root aneurysm or dissection at a bypass temperature of around $31^{\circ} \mathrm{C}$. For those who underwent arch surgery, ARR was performed during cooling. Arch replacement was performed when the circulatory arrest had been established at $24-26{ }^{\circ} \mathrm{C}$ with selective cerebral perfusion through the right axillary artery. The aortic arch and proximal descending aorta were opened. A catheter sheath containing a stent graft of $15 \mathrm{~cm}$ was inserted into the true lumen of the descending aorta. The distal end of a 4-branched artificial vascular graft was anastomosed to the descending aorta containing the intraluminal stented graft. The lower body perfusion was then initiated, and the left carotid artery was reconstructed first. After the anastomosis was finished, selective cerebral perfusion was discontinued and rewarming commenced. Then, the left subclavian artery and the innominate artery were reconstructed. Finally, the proximal end of the prosthetic graft was anastomosed to the artificial ascending aorta.

\section{Follow-up}

All patients who survived surgery were followed up regularly through clinic visits or phone calls. Patients 


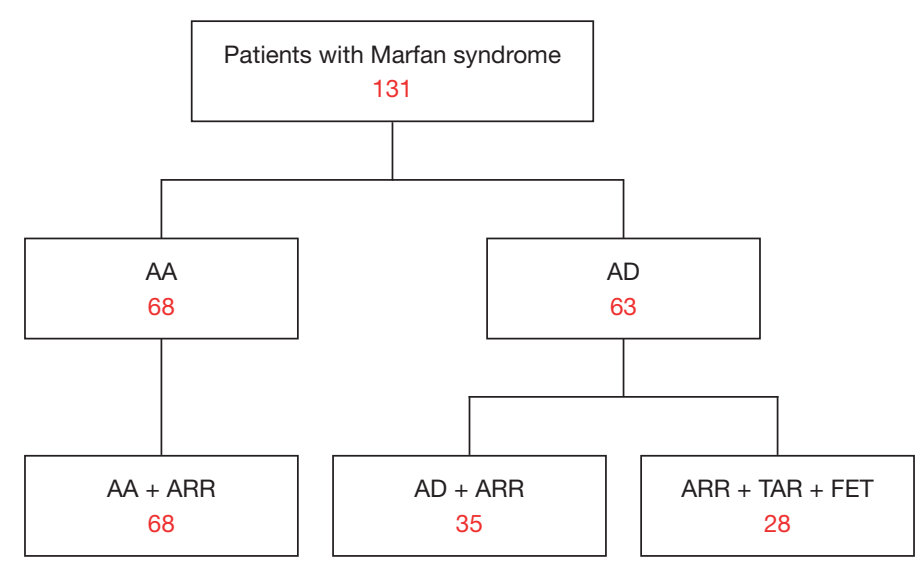

Figure 1 Patients with Marfan syndrome and aortic disease. AA, aortic aneurysm; ARR, aortic root replacement; AD, aortic dissection; TAR, total arch replacement; FET, frozen elephant trunk.

were recommended to undergo computed tomography angiography (CTA) scanning of thoracic and abdominal aorta annually to detect endoleak, thrombosis and obliteration of the false lumen, residual intimal tear or distal new entry, aneurysmal dilatation in the distal aortic segments, and other complications.

\section{Statistical analysis}

The data were analyzed using SPSS for Windows version 26.0 (IBM Corp., Armonk, NY, USA) and plotted in Prism 8.0 software (GraphPad Software, San Diego, CA, USA). The average value of the responses from the other patients was used to fill in the missing value. The KolmogorovSmirnov test was used to evaluate the normal distribution for continuous variables. Continuous data are expressed as the mean \pm standard deviation (SD) and were analyzed with 1-way analysis of variance (ANOVA) followed by least significance difference (LSD) multiple comparisons for normal distribution. For continuous data with a nonnormal distribution, data are expressed as the median (interquartile range Q1, Q3), and the Wilcoxon rank-sum test was used for analysis. Categorical variables are expressed as numbers and percentages, and were analyzed with the chi-square test or Fisher's exact test. Risk factors for early and late mortality or reinterventions were identified with univariate and multivariate analyses and analyzed with Cox regression. Survival and freedom from reoperation were estimated using the Kaplan-Meier method, and intergroup comparisons were made with the log-rank test. All statistical tests were 2 -sided, and only differences with a $\mathrm{P}$ value of less than 0.05 were considered statistically significant.

The primary outcomes were long-term survival and freedom from reoperation. Operative mortality was defined as any death regardless of cause occurring within 30 days of surgery. Variables considered in the analyses included age (years), sex, body mass index (BMI) morbidities, eye disease, family history, diameter of the aortic sinus, surgical treatment, diagnosis, CPB time, cross-clamping time, mechanical ventilation time, and intensive care unit (ICU) time.

\section{Results}

\section{Patients}

Among the 131 patients, 68 patients were diagnosed with $\mathrm{AA}$ and underwent ARR; they were labelled the AA + ARR group. The remaining 63 patients had $\mathrm{AD}$; in 35 of these patients, the dissection originated in and was confined to the ascending aorta, and they underwent ARR (AD + ARR), while the others underwent ARR and total arch replacement combined with FET [ARR + total arch replacement (TAR) + FET] (Figure 1). The mean patient age was $38.36 \pm 11.81$ years (range, 11-65 years), and 27 patients were female (20.61\%). A total of 15 patients $(11.45 \%)$ claimed to have a family history of Marfan syndrome. Based on the interval from symptom onset to surgery, 31 patients $(49.21 \%)$ had acute $\mathrm{AD}(\leq 14$ days), and 32 (50.79\%) had chronic $\mathrm{AD}$ (>14 days). In patients with chronic $\mathrm{AD}$, the mean interval from symptom onset to surgery was 137.13 days (median: 79.00 days; interquartile range, 30.00-219.25 days), and the mean interval from diagnosis to surgery was $6.45 \pm 5.03$ days 
Table 1 Patients baseline characteristics and pre-operation clinical profiles

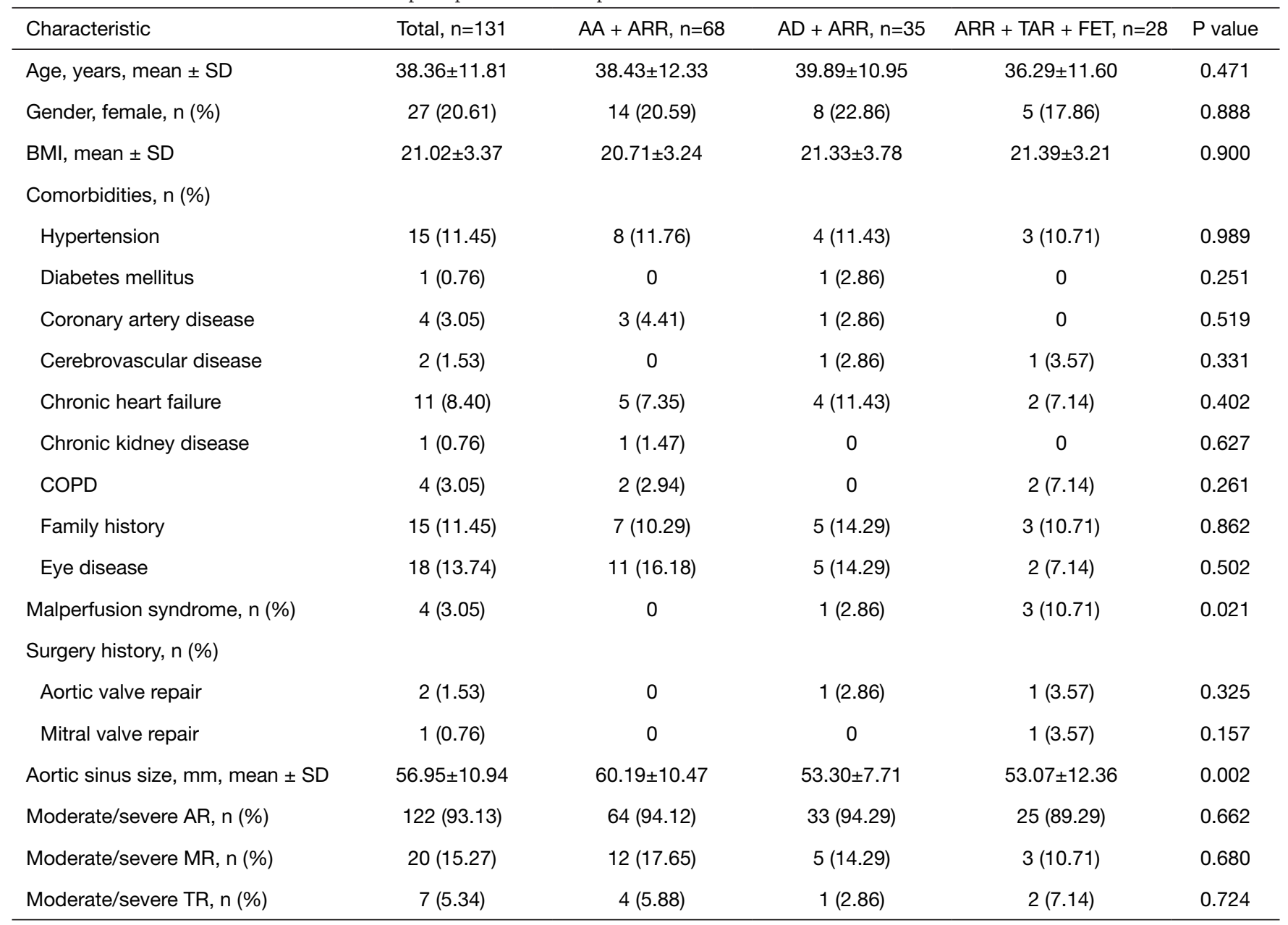

$A R$, aortic valve regurgitation; MR, mitral valve regurgitation; TR, tricuspid valve regurgitation; AA, aortic aneurysm; ARR, aortic root replacement; $A D$, aortic dissection; TAR, total arch replacement; FET, frozen elephant trunk; SD, standard deviation; BMI, body mass index; COPD, chronic obstructive pulmonary disease.

(median: 5.00 days). Severe or moderate AR was present in 122 patients (93.13\%), and mitral valve regurgitation (MR) was present in 20 patients (15.27\%). The aortic sinus diameter averaged $56.95 \pm 10.94 \mathrm{~mm}$ (median: $56.90 \mathrm{~mm}$ ), and the diameter differed significantly between the $\mathrm{AD}$ and AA groups ( $\mathrm{P}=0.002)$ (Table 1). Of note, 16 patients $(12.21 \%)$ had a previous history of Marfan syndrome-related surgery, including aortic valve repair in 2 patients (1.53\%), mitral valve repair in 1 patient $(0.76 \%)$, eye surgery in 4 patients (3.05\%), and orthopedic surgery in 5 patients $(3.82 \%)$. By October 2020, clinical follow-up was complete in 110 of the 125 patients who survived surgery $(88.00 \%)$. The average time was $7.19 \pm 3.34$ years (median: 6.58 years; range, $2.20-17.79$ years).

\section{Operative data}

The entry tear was located in the ascending aorta in 63 patients $(48.09 \%)$. A total of 30 patients had tears involving the aortic arch and the descending aorta, and dissection was limited to the ascending aorta in the other 33 patients. As shown in Table 2, the CPB time was significantly longer in the ARR + TAR + FET group than in the other 2 groups (CPB: $255.96 \pm 46.27$ vs. $198.38 \pm 58.54$ $(\mathrm{AA}+\mathrm{ARR})$ and $207.86 \pm 39.88 \mathrm{~min}(\mathrm{AD}+\mathrm{ARR}) ; \mathrm{P}=0.000)$. The cross-clamping time in the ARR + TAR + FET group did not significantly differ from that in the other groups, and the unilateral selective antegrade cerebral perfusion time was $38.37 \pm 9.54 \mathrm{~min}$. 
Table 2 Intraoperative data

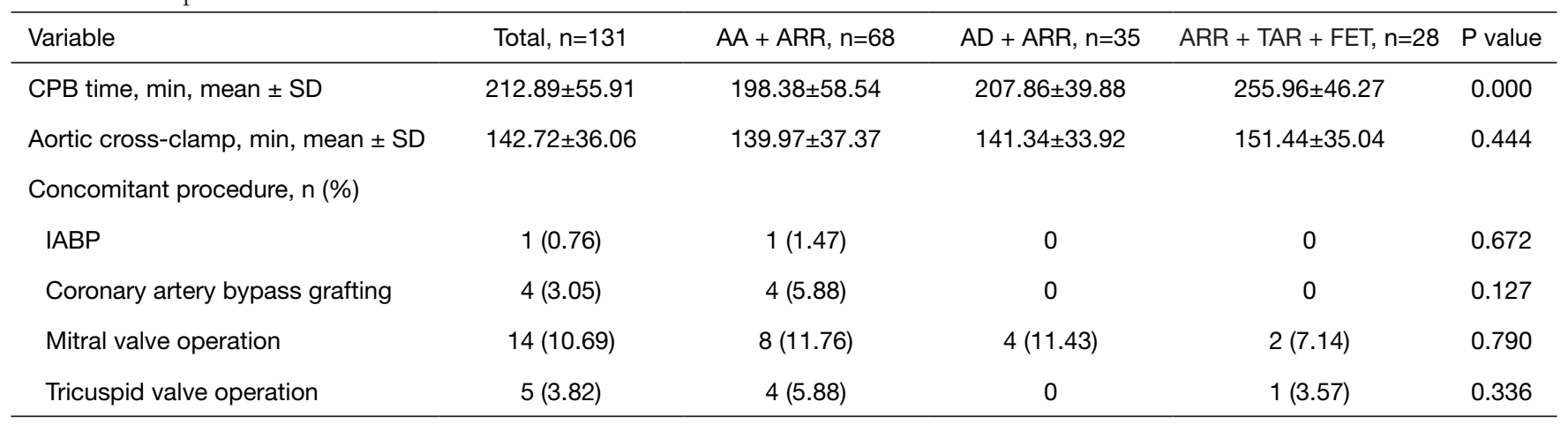

$A A$, aortic aneurysm; ARR, aortic root replacement; AD, aortic dissection; TAR, total arch replacement; FET, frozen elephant trunk; CPB, cardiopulmonary bypass; SD, standard deviation; IABP, intra-aortic balloon pump.

Table 3 Postoperative data and operative mortality

\begin{tabular}{|c|c|c|c|c|c|}
\hline Variable & Total, $n=131$ & $A A+A R R, n=68$ & $A D+A R R, n=35$ & $A R R+T A R+F E T, n=28$ & $P$ value \\
\hline Mechanical ventilation time, days, mean \pm SD & $1.82 \pm 1.28$ & $1.65 \pm 0.99$ & $1.83 \pm 1.46$ & $2.22 \pm 1.60$ & 0.142 \\
\hline Mechanical ventilation time $>3$ days, $n(\%)$ & $13(9.92)$ & $6(8.82)$ & $2(5.71)$ & $5(17.86)$ & 0.252 \\
\hline Respiratory insufficiency, n (\%) & $9(6.87)$ & $3(4.41)$ & $1(2.86)$ & $5(17.86)$ & 0.033 \\
\hline Blood plasma consumption, unit, mean \pm SD & $4.63 \pm 5.05$ & $4.25 \pm 5.15$ & $4.54 \pm 4.60$ & $5.56 \pm 5.36$ & 0.008 \\
\hline ICU stay, days, mean \pm SD & $4.99 \pm 2.41$ & $4.41 \pm 1.58$ & $5.15 \pm 2.68$ & $6.31 \pm 3.28$ & 0.007 \\
\hline Reexploration for bleeding, $\mathrm{n}(\%)$ & $1(0.76)$ & 0 & 0 & $1(3.57)$ & 0.157 \\
\hline Permanent neurological deficit, $n(\%)$ & $5(3.82)$ & $2(2.94)$ & $1(2.86)$ & $2(7.14)$ & 0.585 \\
\hline
\end{tabular}

$\mathrm{AA}$, aortic aneurysm; ARR, aortic root replacement; AD, aortic dissection; TAR, total arch replacement; FET, frozen elephant trunk; SD, standard deviation; ICU, intensive care unit; RRT, renal replacement therapy.

\section{Operative mortality and morbidity}

Operative mortality occurred in 6 patients $(4.58 \%)$, including 3 deaths $(4.41 \%)$ in the AA + ARR group, 1 death $(2.86 \%)$ in the $\mathrm{AD}+\mathrm{ARR}$ group, and 2 deaths in the ARR + TAR + FET group (7.14\%) $(\mathrm{P}=0.718)$. The main cause of death was multi-organ failure. The perioperative rate for permanent neurological deficit occurred in 5 of 6 participant deaths. Early morbidity occurred in 2 patients (1.56\%) who experienced renal failure. Postoperative respiratory insufficiency occurred in 9 patients $(6.87 \%)$ and was most common in the ARR + TAR + FET group (5, 17.86\%; $\mathrm{P}=0.033)$. In addition, red blood cell consumption, blood plasma consumption, and ICU stay were much higher in the ARR + TAR + FET group than in the other groups (Table 3).

\section{Long-term outcomes}

During follow-up, only 6 patients experienced mild bleeding or thromboembolic complications related to anticoagulation therapy.

The freedom from reoperation rate was $88.36 \%$ [95\% confidence interval (CI): $80.32-93.25 \%$ ] at 5 years and $71.75 \%$ (95\% CI: $57.88-81.74 \%$ ) at 10 years (Figure $2 A)$. Late reoperations were required in 22 patients $(17.60 \%)$, but only 10 of these patients (45.45\%) underwent reoperations (Table 4). A total of 7 patients underwent thoracic endovascular aortic repair (TEVAR) for distal AD after ARR. The TEVAR was performed for distal new entry in 7 patients at $0.78,2.26,2.75,2.97,3.00,3.55$, and 13.01 years, respectively. There were 2 patients who underwent TAR 
A
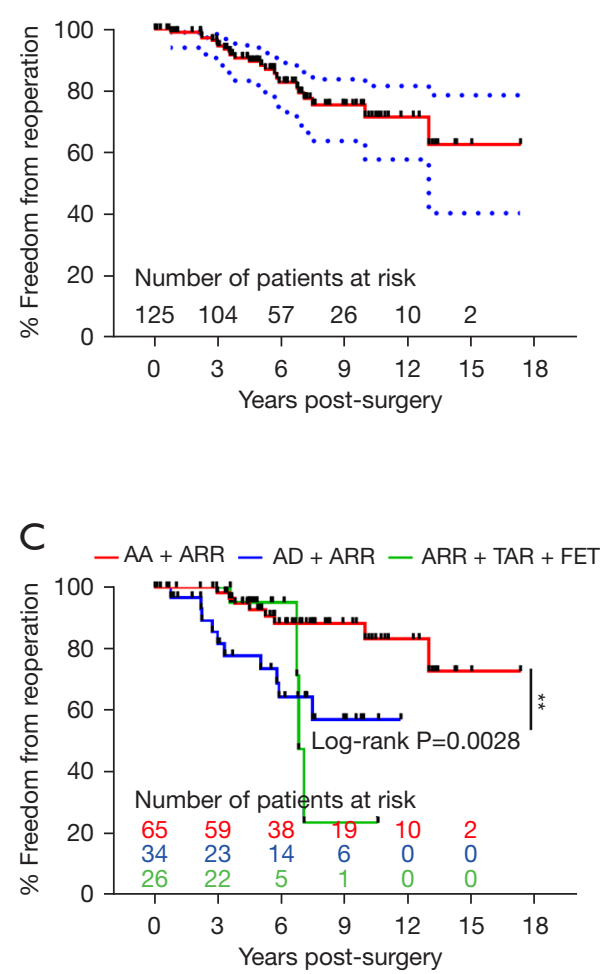

B
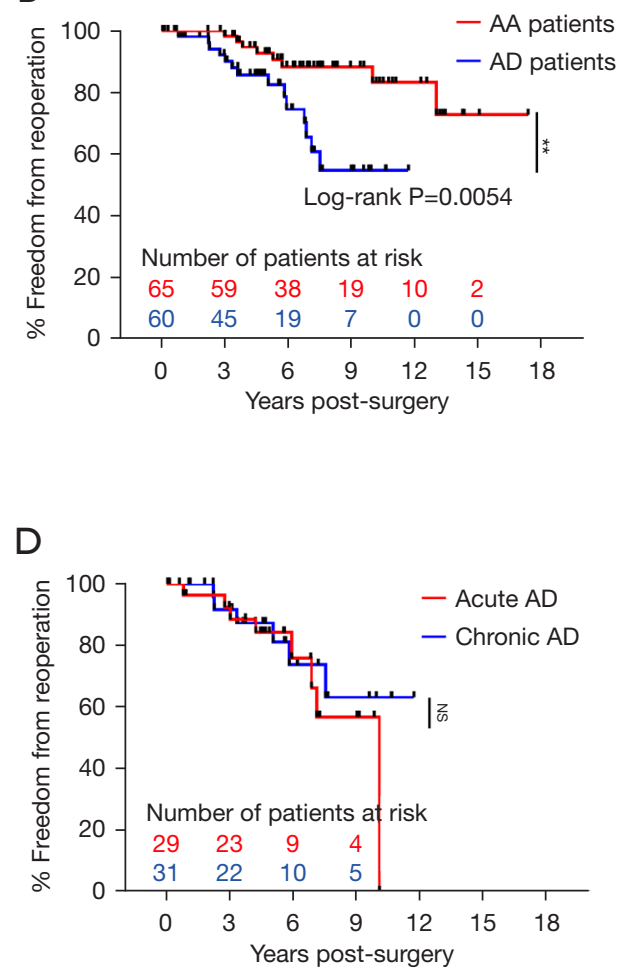

Figure 2 Kaplan-Meier curves of freedom from reoperation according to different groups. (A) Freedom from reoperation of Marfan syndrome patients after ARR. (B) Freedom from reoperation for Marfan syndrome patients and AD compared to those with Marfan syndrome and AA. (C) Long-term freedom from reoperation in subgroups. (D) Freedom from reoperation for acute AD compared to chronic AD in Marfan syndrome patients. AA, aortic aneurysm; AD, aortic dissection; ARR, aortic root replacement; TAR, total arch replacement; FET, frozen elephant trunk.

combined with FET at 5.81 and 10.00 years. Mitral valve replacement and prosthetic aortic valve repair were performed on 1 participant 3.33 years after previous ARR. One participant died of multi-organ dysfunction during the postoperative period, and 1 participant died of distal dissection rupture 3.87 years after TEVAR. One participant was lost to follow-up 1.84 years after reoperation. The remaining 7 patients were alive at a mean $3.57 \pm 2.87$ years (range, $0.10-7.15$ years) after reoperation. Among the other 12 patients, 5 died of distal dissection rupture, and 1 died of heart failure before reoperation. The remaining 6 patients refused to undergo reoperations, among whom only 3 survived. Patients with Marfan syndrome and AD had a higher risk for reoperations than did those with Marfan syndrome and AA [odds ratio $(\mathrm{OR})=3.13 ; 95 \%$ CI: 1.31-7.47; Figure 2B]. The combination of TAR and FET was an alternative for patients with Marfan syndrome and $\mathrm{AD}$ involving the descending aorta in the short term
( $<6$ years) but had a higher risk for reoperation, with a median of 6.84 years of freedom from reoperation (Figure 2C). Additionally, there was no significant difference in freedom from reoperation between acute $\mathrm{AD}$ and chronic $\mathrm{AD}$ patients (Figure 2D).

For the whole cohort, the survival rate was $92.03 \%$ (95\% CI: 83.91-96.15\%) at 5 years and $83.22 \%$ (95\% CI: $71.69-90.37 \%$ ) at 10 years (Figure 3A). Of the 125 hospital survivors, 15 patients were lost to follow-up, and 12 died during subsequent follow-up (Table 5). A total of 6 patients died of distal aortic rupture, 3 died of residual dissection, and 3 died of recurring dissection. A single participant died of renal failure, and another participant died of hemorrhagic shock unrelated to dissection rupture. Another 2 patients died in the postoperative period due to ventricular fibrillation and multi-organ failure. Patients with Marfan syndrome and $\mathrm{AD}$ were shown to have a higher risk for late death than were those with Marfan syndrome and AA 
Table 4 Indication and outcomes of late reoperations

\begin{tabular}{|c|c|c|c|c|c|c|}
\hline Diagnosis & $\begin{array}{c}\text { Surgical } \\
\text { procedures }\end{array}$ & $\begin{array}{c}\text { Years } \\
\text { post-surgery }\end{array}$ & Indication & Intervention & $\begin{array}{l}\text { Survival after } \\
\text { intervention }\end{array}$ & Status \\
\hline$A D$ & ARR & 0.78 & Distal new entry & TEVAR & 2.11 & Alive \\
\hline$A D$ & ARR & 3.33 & $\mathrm{AR}+\mathrm{MR}$ & AVP + MVR & 0.1 & Alive \\
\hline$A D$ & ARR + TAR + TEVAR & 6.86 & Lower limb ischemia & Refused surgery & NA & Lost \\
\hline$A D$ & ARR + TAR + TEVAR & 3.61 & Distal new entry and MR & Refused surgery & 0.60 & Died \\
\hline AA & ARR & 5.27 & $A D$ & Refused surgery & 3.78 & Alive \\
\hline AA & $A R R+M V R$ & 3.84 & $A D$ & NA & NA & Died \\
\hline AA & ARR & 2.97 & $A D$ & TEVAR & 7.15 & Alive \\
\hline AA & ARR & 10.00 & $A D$ & TAR + TEVAR & 0.37 & Alive \\
\hline AA & ARR & 3.55 & $A D$ & TEVAR & 6.97 & Alive \\
\hline$A D$ & ARR & 5.04 & Distal new entry & NA & NA & Died \\
\hline$A D$ & ARR & 2.26 & Distal new entry & TEVAR & 3.87 & Died \\
\hline$A D$ & ARR & 2.22 & $\mathrm{AR}$ and $\mathrm{MR}$ & NA & NA & Died \\
\hline$A D$ & ARR & 7.51 & Distal new entry & NA & NA & Died \\
\hline
\end{tabular}

$A D$, aortic dissection; ARR, aortic root replacement; TEVAR, thoracic endovascular aortic repair; AR, aortic valve regurgitation; MR, mitral valve regurgitation; AVP, aortic valve plasty; MVR, mitral valve replacement; TAR, total arch replacement; NA, not applicable; AA, aortic aneurysm.

$(\mathrm{OR}=4.69 ; 95 \%$ CI: 1.47-14.95; Figure 3B). The TAR plus FET approach had more acceptable results for patients with Marfan syndrome and severe $\mathrm{AD}$ involving the descending aorta than it did for Marfan syndrome patients with AA and was better than ARR alone for Marfan syndrome patients and $\mathrm{AD}(\mathrm{P}>0.05$; Figure $3 \mathrm{C})$. Marfan syndrome patients with acute $\mathrm{AD}$ had a better survival rate than did those with Marfan syndrome and chronic AD (Figure 3D). Although the $\mathrm{P}$ value measured by the log-rank analysis was 0.089 , Cox survival analysis showed that chronic $\mathrm{AD}$ was a risk factor for late survival ( $\mathrm{OR}=5.55$; $95 \% \mathrm{CI}: 1.05-28.96 ; \mathrm{P}=0.042$ ).

\section{Risk factors for late adverse events and reoperations}

Among the variables assessed in the univariate analyses, multivariate analysis identified age $>40$ years as the sole risk factor for early mortality (OR $=14.85$; $95 \%$ CI: 1.65-132.65; $\mathrm{P}=0.016)$. Risk factors for reoperation were age $<30$ years $(\mathrm{OR}=8.45 ; 95 \% \mathrm{CI}: 1.72-41.56 ; \mathrm{P}=0.009)$, and female sex $(\mathrm{OR}=12.24 ; 95 \% \mathrm{CI}: 2.53-59.08 ; \mathrm{P}=0.002)$. Risk factors for late survival were age $<25$ years $(\mathrm{OR}=6.90 ; 95 \% \mathrm{CI}$ : 2.21-21.51; $\mathrm{P}=0.001)$, and female sex $(\mathrm{OR}=2.93 ; 95 \% \mathrm{CI}$ : $1.06-8.10 ; \mathrm{P}=0.039)$. 

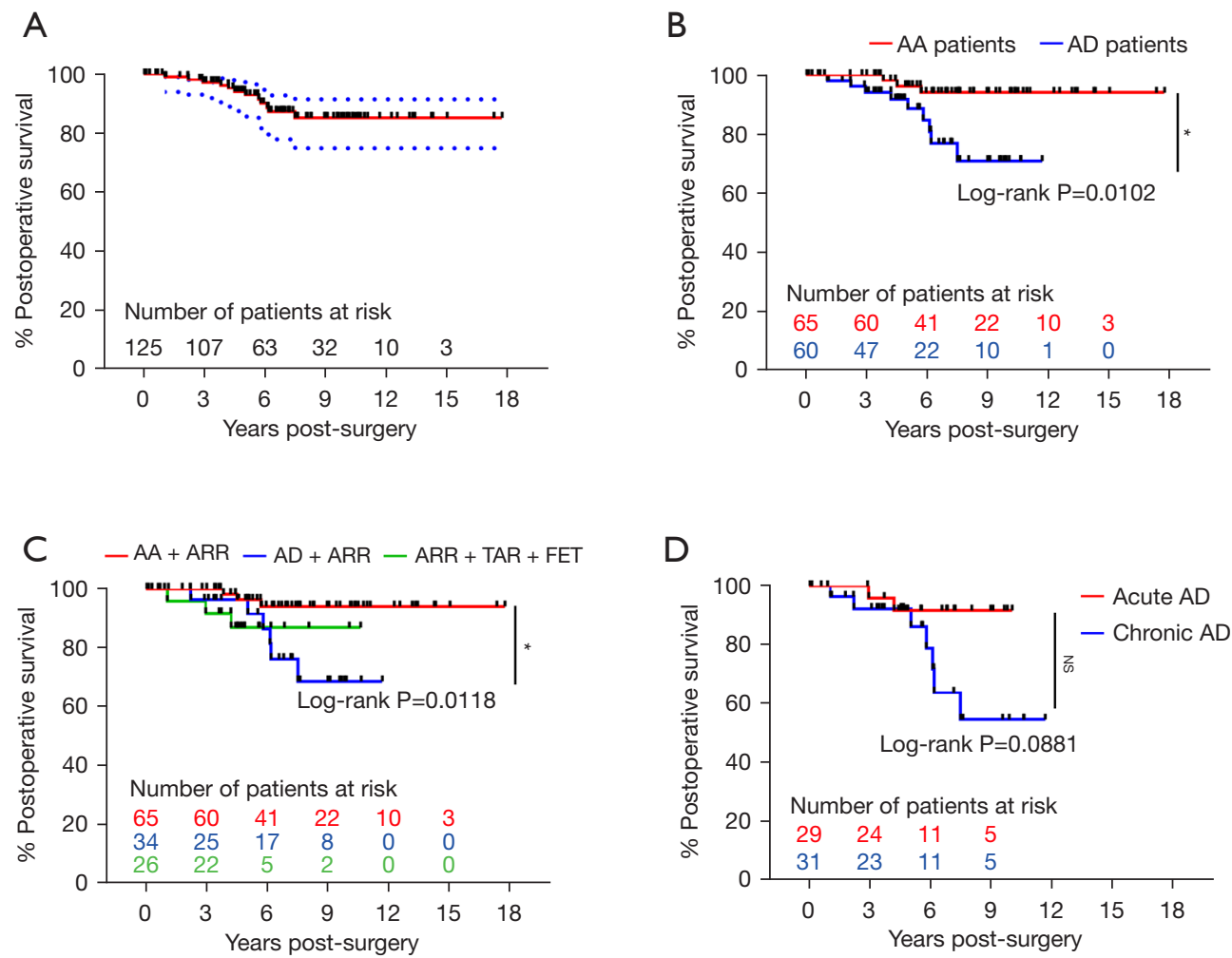

Figure 3 Kaplan-Meier curves of postoperative survival according to different groups. (A) Survival rate of Marfan syndrome patients after ARR. (B) Survival rate for Marfan syndrome patients and AD compared to those with Marfan syndrome and AA. (C) Long-term survival rate in subgroups. (D) Survival rate for acute AD compared to chronic AD in Marfan syndrome patients. AA, aortic aneurysm; AD, aortic dissection; ARR, aortic root replacement; TAR, total arch replacement; FET, frozen elephant trunk.

Table 5 Times, causes, and clues of late death

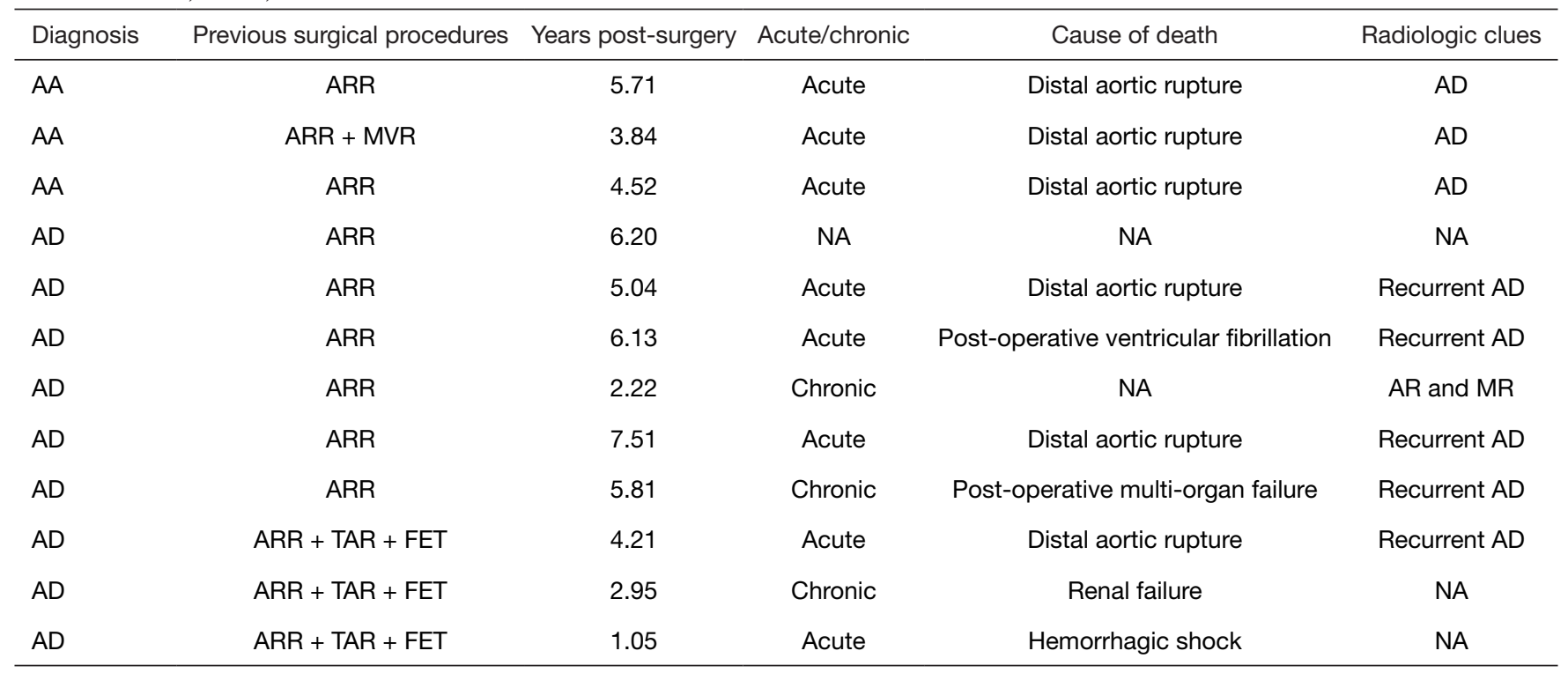

AA, aortic aneurysm; ARR, aortic root replacement; MVR, mitral valve replacement; AD, aortic dissection; NA, not applicable; AR, aortic valve regurgitation; MR, mitral valve regurgitation; TAR, total arch replacement; FET, frozen elephant trunk. 


\section{Discussion}

Several surgical procedures have been developed to treat Marfan syndrome; however, the timing and choice of surgical procedure may have become even more complex and uncertain. Patients with Marfan syndrome who undergo different surgical strategies should undergo further observation (10). Aortic valve sparing root surgery (David procedure) has demonstrated equal outcomes in Marfan syndrome patients relative to ARR, although there are theoretical challenges of greater tissue fragility in patients with Marfan syndrome (4). Previous studies have reported that primary aortic arch prophylactic replacement is not required with a small risk of reinterventions for Marfan syndrome patients undergoing elective root repair (11).

In patients with Marfan syndrome, initial presentation with acute $\mathrm{AD}$ is associated with high mortality and reintervention rates (12). There is a low mortality rate for surgery limited to the aortic root, ascending aorta, and proximal aortic arch for patients with Marfan syndrome presenting with acute $\mathrm{AD}$. New dissection in the residual intact arch after ARR is extremely infrequent (13). Therefore, Marfan syndrome patients with annuloaortic ectasia undergoing ARR are not recommended to undergo prophylactic replacement of the intact arch (13).

Connective tissue disorders such as Marfan syndrome increase the complexity of aortic arch and descending aortic pathology management. Open operation has low rates of morbidity and mortality in the young population with Marfan syndrome (14). However, in one study of Marfan syndrome patients and $\mathrm{AD}, \mathrm{FET}$ appeared to be an efficient therapeutic alternative, and positive aorta remodeling was observed through a delicate approach. Aortic stent grafting may become part of multistage aortic repair, allowing complete treatment of the dissected aorta (15). Both TAR and FET were found to be feasible and efficacious for type A AD following previous ARR in patients with Marfan syndrome (16), while true lumen expansion across the aorta, false lumen, and distal aorta stabilization has been shown to be obtainable through TEVAR $(17,18)$.

In our study, we selected ARR and TAR combined with FET as the appropriate method to treat Marfan syndrome patients with $\mathrm{AD}$, and a satisfactory long-term survival rate was observed. Aggressive surgical intervention is an option available for patients with Marfan syndrome when performing ascending aorta and pre-emptive aortic arch replacement after type A AD (19-21).

Previous reports have demonstrated that more than one-third of patients with Marfan syndrome experience an aortic event, and male patients have significantly more aortic events than do females (22). In our study, we found that female Marfan syndrome patients had a higher risk of mortality and reoperation. The risk of $\mathrm{AD}$ in pregnant women with Marfan syndrome is $1.9 \%$ in the UK (23). Since 2017, our department has successfully treated 3 pregnant women with Marfan syndrome and acute AD. Follow-up is ongoing, and no adverse events have been reported so far.

Fibrillin-1 (FBN1) is the causative gene in the pathogenesis of Marfan syndrome, yet an FBN1 mutation sometimes associates with different phenotypes of Marfan syndrome $(24,25)$. According to ClinVar (https://www.ncbi. nlm.nih.gov/clinvar/), different variation locations in FBN1 exhibit various conditions in patients. Gene arrays from patients with hereditary thoracic $\mathrm{AA}$ and $\mathrm{AD}$ may inform clinical laboratories in the development, interpretation, and subsequent clinical implications of genetic testing for Marfan syndrome patients (26). Reports have shown that Marfan syndrome patients with a haploinsufficient (reduced fibrillin-1 protein levels) mutation are at a higher risk of cardiovascular death and $\mathrm{AD}$ than are patients with abnormal fibrillin-1 protein (27). Worse prognosis, including increased risk for cardiac surgery, $\mathrm{AD}$, and mortality, is related to reduced fibrillin-1 protein levels in Marfan syndrome patients, which demonstrates that genotype impacts phenotypic severity (27). Extensive screening of the whole genome and evaluation of the influence of the FBN1 mutation on fibrillin-1 protein expression have been recommended for Marfan syndrome patients for optimal assessment of prognosis and treatment (27). Previous studies have suggested that patients aged $<35$ years are at higher risk for late distal aortic dilation, reoperation, and death (16). Our studies further corroborated that age is a risk factor for reoperation and death. Age $<30$ years increases the risk for reoperation by 8.45 folds, while age $<25$ years increases the risk for late death by 6.90 folds. Also, for young patients, other connective tissue diseases that need to be considered in the process of differential diagnosis include Ehlers-Danlos syndrome type IV or Loeys-Dietz syndrome, which can have life-threatening cardiovascular manifestations. In our study, all patients fulfilled the Ghent criteria, and the presence of aortic sinus dilation were confirmed in the operation. However, we cannot completely eliminate the possibility of the existence of these 2 diseases without genetic detection methods. We highlight the crucial role of genetic analysis in these patients. 


\section{Limitations}

Our study was a single-center, retrospective investigation with a limited sample size, which was the major challenge to extracting valuable information in the heterogeneous patient cohort. The multivariate regression model and statistical power might have been influenced by the small number of cases. Meanwhile, the rate of follow-up completion was $88 \%$, which is lower than $95 \%$, and thus not sufficiently powerful to be considered representative. Also, we failed to enrol Marfan syndrome patients who underwent valve-sparing ARR as the control group. In recent years, substantial theoretical and practical changes have been made in the treatment of patients with Marfan syndrome, which might have created bias in the estimation of survival and reoperation.

\section{Conclusions}

In Marfan syndrome patients with aortic disease, ARR can be safely performed with low operative mortality, favorable long-term survival, and freedom from reoperation. Young and female patients are at an increased risk for late death and reoperation. To prevent $\mathrm{AD}$, surgical intervention should promptly follow the diagnosis of aortic sinus dilation. These results support the use of ARR in the management of patients with Marfan syndrome.

\section{Acknowledgments}

We would like to thank Dr. J. Jones and Dr. J. Gray from AME Editing Service for their polishing of our manuscript. Funding: This work was supported by the Clinical Research Project Plan of Army Medical University (grant No. 2018XLC3021).

\section{Footnote}

Reporting Checklist: The authors have completed the STROBE reporting checklist. Available at https://dx.doi. org/10.21037/jtd-21-577

Data Sharing Statement: Available at https://dx.doi. org/10.21037/jtd-21-577

Conflicts of Interest: All authors have completed the ICMJE uniform disclosure form (available at https://dx.doi. org/10.21037/jtd-21-577). The authors have no conflicts of interest to declare.

Ethical Statement: The authors are accountable for all aspects of the work in ensuring that questions related to the accuracy or integrity of any part of the work are appropriately investigated and resolved. The study was conducted in accordance with the Declaration of Helsinki (as revised in 2013). The retrospective study was approved by the Medical Ethics Committee of the Second Affiliated Hospital, Army Medical University, Chongqing, China (No. 2020-Yan-122-01) and informed consent was taken from all the patients.

Open Access Statement: This is an Open Access article distributed in accordance with the Creative Commons Attribution-NonCommercial-NoDerivs 4.0 International License (CC BY-NC-ND 4.0), which permits the noncommercial replication and distribution of the article with the strict proviso that no changes or edits are made and the original work is properly cited (including links to both the formal publication through the relevant DOI and the license). See: https://creativecommons.org/licenses/by-nc-nd/4.0/.

\section{References}

1. Erbel R, Aboyans V, Boileau C, et al. 2014 ESC Guidelines on the diagnosis and treatment of aortic diseases:

Document covering acute and chronic aortic diseases of the thoracic and abdominal aorta of the adult. The Task Force for the Diagnosis and Treatment of Aortic Diseases of the European Society of Cardiology (ESC). Eur Heart J 2014;35:2873-926.

2. Gott VL, Greene PS, Alejo DE, et al. Replacement of the aortic root in patients with Marfan's syndrome. N Engl J Med 1999;340:1307-13.

3. Price J, Magruder JT, Young A, et al. Long-term outcomes of aortic root operations for Marfan syndrome: A comparison of Bentall versus aortic valve-sparing procedures. J Thorac Cardiovasc Surg 2016;151:330-6.

4. David TE, David CM, Manlhiot C, et al. Outcomes of Aortic Valve-Sparing Operations in Marfan Syndrome. J Am Coll Cardiol 2015;66:1445-53.

5. Shrestha ML, Beckmann E, Abd Alhadi F, et al. Elective David I Procedure Has Excellent Long-Term Results: 20-Year Single-Center Experience. Ann Thorac Surg 2018;105:731-8.

6. Judge DP, Dietz HC. Marfan's syndrome. Lancet 2005;366:1965-76. 
7. Ma WG, Zhu JM, Zheng J, et al. Sun's procedure for complex aortic arch repair: total arch replacement using a tetrafurcate graft with stented elephant trunk implantation. Ann Cardiothorac Surg 2013;2:642-8.

8. Sun L, Qi R, Zhu J, et al. Total arch replacement combined with stented elephant trunk implantation: a new "standard" therapy for type a dissection involving repair of the aortic arch? Circulation 2011;123:971-8.

9. Sun L, Qi R, Chang Q, et al. Surgery for marfan patients with acute type a dissection using a stented elephant trunk procedure. Ann Thorac Surg 2008;86:1821-5.

10. Groenink M, Koolbergen DR. Surgical treatment of Marfan syndrome and related disorders is all about dealing with uncertainties. Heart 2018;104:454-5.

11. Schoenhoff FS, Kadner A, Czerny M, et al. Should aortic arch replacement be performed during initial surgery for aortic root aneurysm in patients with Marfan syndrome? Eur J Cardiothorac Surg 2013;44:346-51; discussion 351.

12. Schoenhoff FS, Jungi S, Czerny M, et al. Acute aortic dissection determines the fate of initially untreated aortic segments in Marfan syndrome. Circulation 2013;127:1569-75.

13. Tagusari O, Ogino H, Kobayashi J, et al. Should the transverse aortic arch be replaced simultaneously with aortic root replacement for annuloaortic ectasia in Marfan syndrome? J Thorac Cardiovasc Surg 2004;127:1373-80.

14. Waterford SD, Moon MR. Stent grafting in Marfan syndrome? We are not convinced. J Thorac Cardiovasc Surg 2018;156:1773-5.

15. Pellenc Q, Girault A, Roussel A, et al. Optimising Aortic Endovascular Repair in Patients with Marfan Syndrome. Eur J Vasc Endovasc Surg 2020;59:577-85.

16. Chen Y, Ma WG, Zheng J, et al. Total arch replacement and frozen elephant trunk for type A aortic dissection after Bentall procedure in Marfan syndrome. J Thorac Dis 2018;10:2377-87.

17. Chen Y, Ma WG, Zhi AH, et al. Fate of distal aorta after frozen elephant trunk and total arch replacement for type A aortic dissection in Marfan syndrome. J Thorac

Cite this article as: Zhu Y, Jian Z, Ma R, Wang Y, Xiao Y. Long-term outcomes after aortic root replacement for patients with Marfan syndrome. J Thorac Dis 2021;13(12):6779-6789. doi: $10.21037 /$ jtd-21-577
Cardiovasc Surg 2019;157:835-49.

18. Czerny M, Schmidli J, Adler S, et al. Current options and recommendations for the treatment of thoracic aortic pathologies involving the aortic arch: an expert consensus document of the European Association for CardioThoracic surgery (EACTS) and the European Society for Vascular Surgery (ESVS). Eur J Cardiothorac Surg 2019;55:133-62.

19. Rylski B, Bavaria JE, Beyersdorf F, et al. Type A aortic dissection in Marfan syndrome: extent of initial surgery determines long-term outcome. Circulation 2014;129:1381-6.

20. Milleron O, Arnoult F, Delorme G, et al. Pathogenic FBN1 Genetic Variation and Aortic Dissection in Patients With Marfan Syndrome. J Am Coll Cardiol 2020;75:843-53.

21. Cañadas V, Vilacosta I, Bruna I, et al. Marfan syndrome. Part 2: treatment and management of patients. Nat Rev Cardiol 2010;7:266-76.

22. Groth KA, Stochholm K, Hove H, et al. Aortic events in a nationwide Marfan syndrome cohort. Clin Res Cardiol 2017;106:105-12.

23. Cauldwell M, Steer PJ, Curtis SL, et al. Maternal and fetal outcomes in pregnancies complicated by Marfan syndrome. Heart 2019;105:1725-31.

24. Turner CL, Emery H, Collins AL, et al. Detection of 53 FBN1 mutations (41 novel and 12 recurrent) and genotype-phenotype correlations in 113 unrelated probands referred with Marfan syndrome, or a related fibrillinopathy. Am J Med Genet A 2009;149A:161-70.

25. Li Y, Xu J, Chen M, et al. A FBN1 mutation association with different phenotypes of Marfan syndrome in a Chinese family. Clin Chim Acta 2016;460:102-6.

26. Renard M, Francis C, Ghosh R, et al. Clinical Validity of Genes for Heritable Thoracic Aortic Aneurysm and Dissection. J Am Coll Cardiol 2018;72:605-15.

27. Franken R, Groenink M, de Waard V, et al. Genotype impacts survival in Marfan syndrome. Eur Heart J 2016;37:3285-90. 